\title{
Percepção de dermatologistas sobre a influência da psoríase na qualidade de vida dos pacientes
}

\section{Perception of dermatologists on the influence of psoriasis on quality of life of patients}

\section{Percepción de los dermatólogos sobre el impacto de la psoriasis en la calidad de vida de los pacientes}

\author{
Jhenifer Mellissa de Souza ${ }^{1}$, ORCID 0000-0003-2003-8738 \\ Natalia Aparecida de Godoy ${ }^{2}$, ORCID 0000-0002-1093-1437 \\ Camila Fernandes Pollo ${ }^{3}$, ORCID 0000-0003-0264-5841 \\ Hélio Amante Miot ${ }^{4}$, ORCID 0000-0002-2596-9294 \\ Silmara Meneguin 5, ORCID 0000-0003-3853-5134
}

12345 Faculdade de Medicina de Botucatu. Brasil

Recebido: 09/03/2021

Aceito: 14/02/2021

\begin{abstract}
Resumo: Objetivo: Conhecer como a psoríase interfere na qualidade de vida dos pacientes na percepção de dermatologistas e identificar estratégias para melhoria e reorganização da assistência. Métodos: Pesquisa exploratória e descritiva, realizada em hospital público do interior de São Paulo, no período de dezembro de 2017 a março de 2018, com seis dermatologistas. As entrevistas foram transcritas e analisadas utilizando-se a estratégia metodológica do Discurso do Sujeito Coletivo. Resultados: Psoríase é uma doença que inflige prejuízo à qualidade de vida por estar associada a transtornos psicossociais, estigma, vergonha e perda da autoestima. Reduzir lesões e sintomas, melhorar a aparência, disponibilizar medicamento eficaz e oferecer atendimento humanizado são aspectos fundamentais para melhoria da qualidade de vida destes pacientes. Conclusão: Para os profissionais dermatologistas, a psoríase é uma doença crônica, que interfere na qualidade de vida dos pacientes, em virtude do preconceito sentido, dos transtornos psicossociais e sentimentos negativos que afloram, bem como das dificuldades sociais enfrentadas.
\end{abstract}

Palavras-chave: psoríase; qualidade de vida; dermatologia; enfermagem.

Abstract: Objective: to know how psoriasis affects the quality of life of patients in the perception of dermatologists and identify strategies for improving it and reorganizing care. Methods: Descriptive, exploratory study research conducted in a public hospital in the countryside of São Paulo, from December 2017 to March 2018, with six dermatologists. The interviews were transcribed and analyzed using the methodological strategy of the Collective Subject Discourse. 
Results: Psoriasis is a disease, which affects the quality of life because it is associated with psychosocial disorders, shame, stigma and loss of self-esteem. Reducing injuries and symptoms, improving appearance, having an effective medication coupled with humanized care are fundamental for improving the quality of life of these patients. Conclusion: It was observed that for dermatologists, psoriasis is a chronic disease that interferes with the quality of life, due to the prejudice felt by patients, the psychosocial disorders and negative feelings that arise, as well as the social difficulties faced, by them.

Keywords: psoriasis; quality of life; dermatology; nursing.

Resumen: Objetivos: conocer cómo la psoriasis afecta la calidad de vida de los pacientes en la percepción de los dermatólogos e identificar estrategias para mejorarla y reorganizar la atención. Métodos: Investigación descriptiva y exploratoria realizada en un hospital público del interior de São Paulo, de diciembre de 2017 a marzo de 2018, con seis dermatólogos. Las entrevistas fueron transcritas y analizadas utilizando la estrategia metodológica del Discurso Colectivo del Sujeto. Resultados: En opinión de los participantes, la psoriasis es una enfermedad que daña la calidad de vida por estar asociada a trastornos psicosociales, estigma, vergüenza y pérdida de la autoestima. Reducir lesiones y síntomas, mejorar la apariencia, disponer de una medicación eficaz vinculada a la atención humanizada son fundamentales para mejorar la calidad de vida de estos pacientes. Conclusión: Se observó que, para los dermatólogos, la psoriasis es una enfermedad crónica que interfiere con la calidad de vida, debido al prejuicio sentido, los trastornos psicosociales y sentimientos negativos que surgen, así como las dificultades sociales que enfrentan los pacientes.

Palabras clave: psoriais; calidad de vida; dermatología; enfermería.

Correspondência: Silmara Meneguin - e-mail: s.meneguin@unesp.br

\section{Introdução}

Psoríase é uma doença inflamatória crônica da pele e das articulações, imunomediada, influenciada por fatores genéticos e ambientais, que acomete pacientes de todas as faixas etárias e de ambos os sexos.(1-3) Caracteriza-se pelo aparecimento de placas eritemato-descamativas com crostas brancas sobrepostas, de tamanhos variados, discoides ou já círculos completos ou incompletos.(4-5)

Por regra geral, a evolução da psoríase se prolonga por muitos anos. No entanto, observase a frequente modificação na sua forma e até mesmo o seu desaparecimento, seguidos da recidiva da doença.(6)

A despeito do grande impacto causado por essa dermatose na qualidade de vida, apenas nos últimos 20 anos houve preocupação dos profissionais de saúde com as repercussões sistêmicas causadas na vida dos pacientes.(7) 
Para a Organização Mundial da Saúde (OMS), qualidade de vida é conceituada como "a percepção do indivíduo quanto a sua posição na vida, no contexto da cultura e do sistema de valores nos quais vive e em relação a seus objetivos, expectativas, padrões e preocupações".(8) Embora seja um conceito abrangente e amplo, reflete os aspectos subjetivos e multidimensionais que permeiam o constructo.(9)

Pessoas com doenças crônicas podem ter a qualidade de vida alterada, uma vez que precisam modificar hábitos e desenvolver processos adaptativos à doença. Além dos impactos diretos na QV, as dermatoses podem promover agravos psicológicos como alterações na autoimagem, prejuízos na autoestima e nas interações sociais, e desencadear sintomas de depressão e ansiedade.(10-11) Essas implicações têm contribuído para despertar o interesse de pesquisadores que estudam as repercussões na QV provocadas por doenças crônicas como hipertensão, diabetes, doenças reumatológicas e cardiovasculares.(12-13)

Os instrumentos que utilizam parâmetros psicométricos de mensuração da QV podem auxiliar em decisões que envolvem, por exemplo, a administração ou não de medicamentos de alto custo ou de alto risco. A utilização de medidas simples de avaliação de qualidade de vida é algo bem aceito pelos pacientes que desejam expressar suas ansiedades e preocupações.(6)

No entanto, nota-se uma escassez de estudos que avaliam a percepção dos especialistas em dermatologia em relação à qualidade de vida dos pacientes portadores de psoríase, uma vez que a maioria dos estudos avalia qualidade de vida sob a ótica do paciente.(14-15) Diante do exposto, a proposta deste estudo foi responder a seguinte questão norteadora: Qual a influência da psoríase na QV dos pacientes, na percepção de médicos dermatologistas? Como proporcionar QV para esses pacientes? Como essa assistência poderia ser melhorada?

Para preencher essa lacuna do conhecimento, o presente estudo buscou conhecer como a psoríase interfere na qualidade dos pacientes na percepção dos dermatologistas e identificar estratégias para melhoria e reorganização da assistência.

\section{Métodos}

Trata-se de um estudo exploratório e descritivo, que utilizou a técnica do Discurso do Sujeito Coletivo (DSC), fundamentada na teoria das Representações Sociais. O DSC busca responder e conhecer os pensamentos, representações, crenças e valores de uma coletividade sobre determinado tema mediante a aplicação de métodos científicos.(16)

Na pesquisa empírica, o pensamento, materializado sob forma de discurso, é uma variável qualitativa. Mas sendo esse pensamento coletivo, configura-se também como uma variável quantitativa, na medida em que expressa as opiniões compartilhadas pelos indivíduos.(17)

O estudo foi realizado no ambulatório de dermatologia de uma instituição pública, universitária, localizada em um município do interior do estado de São Paulo. Estabeleceu-se como critérios de inclusão: ser médico especialista em dermatologia, de ambos os sexos, ser membro da Sociedade Brasileira de Dermatologia e prestar atendimento no local da pesquisa. Inicialmente, 
fez-se convite aos especialistas para participarem do estudo e a inclusão dos mesmos deu-se somente após a leitura e assinatura do Termo de Consentimento Livre e Esclarecido.

Os dados foram coletados por meio de entrevistas semiestruturadas, realizadas individualmente em ambiente privativo, no período de dezembro de 2017 a março de 2018. Utilizou-se um roteiro de entrevista semiestruturado, elaborado pelos pesquisadores, constituído por três questões norteadoras. Tais perguntas foram aplicadas a seis médicos dermatologistas que prestam atendimento a pacientes com o diagnóstico de psoríase no ambulatório de dermatologia da instituição onde a pesquisa foi realizada, as saber: "Na sua opinião, psoríase interfere na qualidade de vida dos pacientes? Se sim, como? Na sua opinião, o que significa proporcionar qualidade de vida para pacientes com psoríase? Como a qualidade de vida destes pacientes poderia ser melhorada?" Ao término das entrevistas, as falas foram transcritas e submetidas à análise manual por um dos pesquisadores com experiência em operacionalizar a estratégia metodológica do Discurso do Sujeito Coletivo (DSC).

Os passos metodológicos desta técnica, que devem ser seguidos desde a obtenção das entrevistas até a síntese dos discursos, incluem: leitura do conjunto dos depoimentos coletados nas entrevistas; leitura da resposta a cada pergunta em particular, marcando as expressões-chave selecionadas; identificação das ideias centrais de cada resposta; análise de todas as expressõeschave e ideias centrais, agrupando as semelhanças em conjuntos homogêneos; identificação e nomeação da ideia central do conjunto homogêneo, que será uma síntese das ideias centrais de cada discurso; construção dos discursos do sujeito coletivo após a identificação das ideias centrais e expressões-chave que nomearam os referidos discursos do sujeito coletivo.(16)

Neste estudo, optou-se por atribuir a identificação dos depoimentos dos entrevistados pela letra "M" (entrevistado) seguida da numeração que lhes foi atribuída na transcrição das entrevistas.

O projeto foi aprovado pelo Comitê de Ética em Pesquisa da Faculdade de Medicina de Botucatu (FMB), sob n 2.392.601, conforme Resolução nº 466/2012 do Conselho Nacional de Saúde.(18)

\section{Resultados}

A amostra foi constituída por seis médicos dermatologistas. Após a transcrição das entrevistas, foram identificadas as ideias centrais e as expressões-chave e organizados os discursos dos três temas, que emergiram das questões norteadoras da entrevista. O Quadro 1 sintetiza as ideias centrais e o DSC referente ao primeiro tema: Impacto da psoríase na qualidade de vida. 
Quadro 1 - Síntese das ideias centrais e discurso do sujeito coletivo referente ao tema "Impacto da psoríase na qualidade de vida".

\begin{tabular}{|c|c|}
\hline Ideias Centrais & DSC \\
\hline Aparência & $\begin{array}{l}\text { Acredito que a qualidade de vida depende muito } \\
\text { do paciente, do grau de acometimento, da } \\
\text { gravidade da doença e, principalmente, da } \\
\text { estética. É também é influenciada pelos aspectos } \\
\text { físicos, como coceira, dor, fissuras e } \\
\text { comprometimento articular. As escamas também } \\
\text { incomodam, ficam nas roupas e as pessoas julgam } \\
\text { que é descuido pessoal }(M 1, M 3, M 4, M 5) \text {. }\end{array}$ \\
\hline $\begin{array}{c}\text { Informação equivocada sobre a } \\
\text { doença }\end{array}$ & $\begin{array}{l}\text { Como a gente acaba atendendo pacientes com } \\
\text { baixo nível de escolaridade, a preocupação com a } \\
\text { transmissão da doença prevalece e interfere em } \\
\text { muitos aspectos da vida, pois se sentem } \\
\text { incomodados pensando no que os outros irão } \\
\text { pensar, por acharem que é uma doença } \\
\text { contagiosa }(\mathrm{M} 2, \mathrm{M} 4) \text {. }\end{array}$ \\
\hline Doença estigmatizante & $\begin{array}{l}\text { Isso contribui para que o paciente que esteja com } \\
\text { lesões de pele se esconda ou esconda as lesões, o } \\
\text { que afeta o convívio social, familiar e contribui } \\
\text { para o estigma da doença (M3, M4, M5 M6). }\end{array}$ \\
\hline $\begin{array}{l}\text { Transtornos psicossociais } \\
\text { (Ansiedade/depressão) }\end{array}$ & $\begin{array}{l}\text { Na minha opinião, a psoríase pode desencadear } \\
\text { sintomas de ansiedade em alguns pacientes, fato } \\
\text { esse que pode levar à piora das lesões }(M 4, M 6) \text {. }\end{array}$ \\
\hline $\begin{array}{c}\text { Comprometimento das atividades } \\
\text { profissionais }\end{array}$ & $\begin{array}{l}\text { Psoríase também interfere nas relações } \\
\text { emocionais, sentimentais, profissionais, o que } \\
\text { contribui para que alguns pacientes solicitem } \\
\text { benefícios secundários, por se afastar do } \\
\text { trabalho, por conta da lesão }(\mathrm{M} 5, \mathrm{M6}) \text {. }\end{array}$ \\
\hline $\begin{array}{l}\text { Perda da autoestima } \\
\text { Sentimentos negativos }\end{array}$ & $\begin{array}{l}\text { Além de tudo, a psoríase, por ser uma doença } \\
\text { crônica, acaba contribuindo para a perda da } \\
\text { autoestima, o que pode levar, a longo prazo, a um } \\
\text { sentimento de impotência, incapacidade, } \\
\text { desesperança e até mesmo depressão }(M 1, M 2 \text {, } \\
\text { M3). }\end{array}$ \\
\hline
\end{tabular}

O Quadro 2 apresenta as ideias centrais e os discursos referentes ao segundo tema: Como proporcionar qualidade de vida para pacientes com psoríase. 
Quadro 2 - Síntese das ideias centrais e discurso do sujeito coletivo referente ao tema "Como proporcionar qualidade de vida para pacientes com psoríase".

\begin{tabular}{|c|c|}
\hline Ideias Centrais & DSC \\
\hline Reduzindo lesões e sintomas & $\begin{array}{l}\text { A resposta certa é curando a doença, mas, } \\
\text { infelizmente, não temos a cura. Isso depende } \\
\text { muito de cada paciente, às vezes o que é qualidade } \\
\text { de vida para um não é pra o outro. } \\
\text { Proporcionar qualidade de vida, para mim, seria } \\
\text { você ter maior redução possível no número de } \\
\text { lesões e melhora da aparência, podendo oferecer } \\
\text { um tipo de tratamento aceitável, com o mínimo de } \\
\text { efeito colateral, e que resulte em um menor } \\
\text { número de lesões. O mais importante é o paciente } \\
\text { se ver sem lesões, estar bem de saúde, mas isso é } \\
\text { sempre muito difícil }(M 1, M 2, M 4, M 5) \text {. }\end{array}$ \\
\hline $\begin{array}{l}\text { Compreendendo o impacto da doença na vida } \\
\text { do paciente }\end{array}$ & $\begin{array}{l}\text { Na minha concepção, eu proporciono qualidade } \\
\text { de vida quando eu entendo em que proporção a } \\
\text { doença o afeta. E isso não significa que ele fique } \\
\text { livre da doença, mas que ele fique um pouco } \\
\text { melhor, com alívio do prurido, com a melhora no } \\
\text { aspecto das lesões e, principalmente, com a } \\
\text { melhora das áreas onde a lesão é mais exposta, } \\
\text { mais visivel, proporcionando facilitar o convivio } \\
\text { social }(M 1, M 3, M 4, M 6) \text {. }\end{array}$ \\
\hline Esclarecendo o paciente sobre a doença & $\begin{array}{l}\text { É nossa função também prestar esclarecimento } \\
\text { aos pacientes sobre a doença, porque muitos } \\
\text { acham que é transmissível, que pode se tornar um } \\
\text { câncer, e informar que a Medicina está se } \\
\text { esforçando para conseguir melhorias e e } \\
\text { tratamento mais eficientes }(M 1, M 3, M 4, M 6) \text {. }\end{array}$ \\
\hline Humanizando o atendimento & $\begin{array}{l}\text { Se conseguirmos proporcionar um bom } \\
\text { acolhimento, um bom atendimento e deixá-lo } \\
\text { seguro de que estaremos oferecendo o nosso } \\
\text { melhor, acredito que poderemos melhorar o } \\
\text { impacto na } Q V(M 2, M 3, M 5) \text {. }\end{array}$ \\
\hline Compreendendo as necessidades do paciente & $\begin{array}{l}\text { Nós, dermatologistas, temos que ter } \\
\text { sensibilidade para entender a necessidade de } \\
\text { cada paciente, perguntar se esse paciente está } \\
\text { dormindo, como está a vida amorosa, se está } \\
\text { tendo prazer nas coisas, enfim, e não somente } \\
\text { nos preocuparmos em tratar a psoríase (M1, } \\
\text { M2, M3, M4). }\end{array}$ \\
\hline
\end{tabular}


O Quadro 3 apresenta as ideias centrais e os discursos referentes ao terceiro tema: Intervenções para melhorar a qualidade de vida de pacientes com psoríase.

Quadro 3 - Síntese das ideias centrais e discurso do sujeito coletivo referente ao tema "Intervenções para melhorar a qualidade de vida de pacientes com psoríase".

\begin{tabular}{|c|c|}
\hline Ideias Centrais & DSC \\
\hline Reduzir lesões e sintomas & $\begin{array}{l}\text { Eu acho que se contribuirmos para a redução do } \\
\text { número de lesões, das dores, da coceira, isso, sem } \\
\text { dúvida, vai impactar de uma forma positiva na } \\
\text { qualidade de vida (M2, M3, M4). }\end{array}$ \\
\hline Esclarecer a população e os pacientes & $\begin{array}{l}\text { Informar a população, a família e o próprio } \\
\text { paciente talvez seja um recurso terapêutico } \\
\text { que diminua o estigma da doença }(M 1, M 2 \text {, } \\
\text { M4, M5, M6). }\end{array}$ \\
\hline Acolhimento & $\begin{array}{l}\text { Além do mais, devemos também acolher o } \\
\text { paciente e entender a representação da } \\
\text { doença na vida dele }(M 2, M 3) \text {. }\end{array}$ \\
\hline $\begin{array}{l}\text { Tentar proporcionar atendimento } \\
\text { multidisciplinar }\end{array}$ & $\begin{array}{l}\text { Toda doença crônica, de forma geral, } \\
\text { consome nossa saúde mental. Então, acho que } \\
\text { precisamos dar uma atenção especial a isso } \\
\text { também. Se pudéssemos contar com um } \\
\text { tratamento multidisciplinar, com } \\
\text { nutricionistas, cardiologistas e psicólogos, } \\
\text { certamente poderíamos oferecer uma } \\
\text { qualidade de vida melhor a esses pacientes } \\
(\mathrm{M} 1, \mathrm{M} 2, \mathrm{M4}, \mathrm{M5}, \mathrm{M} 6) \text {. }\end{array}$ \\
\hline Oferecer tratamentos coadjuvantes & $\begin{array}{l}\text { Oferecer cremes, hidratantes, não somente a } \\
\text { medicação que existe de alto custo, mas os } \\
\text { coadjuvantes. Tudo isso vai cooperar para } \\
\text { que ele seja recolocado na sociedade, } \\
\text { resultando em uma melhor qualidade de vida } \\
(\mathrm{M} 3, \mathrm{M} 5, \mathrm{M6}) \text {. }\end{array}$ \\
\hline
\end{tabular}

\section{Discussão}

As dermatoses prejudicam a autoimagem e têm grande potencial para desencadear processos que afetam a autoestima. Além disso, contribuem para intensificar o sentimento de rejeição provocado pelas doenças de pele e podem desencadear sentimentos negativos em relação à imagem corporal e receio diante de possível exposição corporal. $(10,19)$

Aspectos observados nas falas dos especialistas entrevistados sugerem que a percepção dos pacientes em relação à aparência física está associada a um maior prejuízo nas atividades cotidianas e exclusão social, o que pode comprometer as atividades diárias e a qualidade de vida 
de modo geral. Estudos mostram que indivíduos acometidos pela psoríase em todo o mundo vivenciam a estigmatização social, muitas vezes relacionada ao trabalho, à discriminação pela sociedade, além de dor, prurido e sangramento.(20-21)

Pesquisa qualitativa realizada na Dinamarca com pacientes que apresentam psoríase evidenciou que eles desenvolvem estratégias para evitar a exposição pública em ocasiões sociais em decorrência da estigmatização da doença.(22) O desconforto gerado pela doença contribui para que evitem outras pessoas ou camuflem as lesões, o que pode favorecer a perda da autoestima e até mesmo desencadear sintomas de ansiedade e depressão.(23)

De acordo com os participantes deste estudo, os sentimentos negativos em relação à doença e o aspecto das lesões também podem interferir nas atividades laborais e, por isso, alguns buscam obter benefícios secundários decorrentes do adoecimento, como o afastamento do trabalho. Estudo desenvolvido na Alemanha mostrou que pacientes portadores de psoríase perdiam, em média, 4,9 dias de trabalho por ano em virtude da doença.(24)

Autores ressaltam a presença de diversas variáveis que podem impactar na vida dos pacientes com psoríase. Elas incluem características de personalidade, caráter, valores, situação de vida, significados atribuídos à vida e atitudes da sociedade, que variam de pessoa para pessoa.(25)

Os dados coletados no estudo em tela mostram que proporcionar melhor qualidade de vida para os pacientes demanda acolhimento e sensibilidade da equipe para entender a necessidade de cada um, de forma individualizada. Além disso, foi enfatizada a necessidade de fornecer esclarecimentos aos pacientes e à sociedade, para que possam compreender melhor a representação dessa doença na vida dos indivíduos acometidos. Na perspectiva dos entrevistados, proporcionar tratamento adequado e reduzir os efeitos colaterais provocados pelos medicamentos também pode contribuir para a melhoria da QV. A literatura tem destacado terapias específicas, especialmente com uso de medicamentos biológicos, que possibilitam estabilização total ou parcial da doença.(26-27)

O cuidado de pacientes com psoríase não requer apenas o tratamento das lesões e das implicações articulares, mas, também, a identificação e o tratamento das comorbidades já existentes ou que possam vir a desenvolver. Isso inclui atentar para eventuais doenças cardiovasculares, metabólicas e psicológicas, com uma equipe multiprofissional (28)

Em estudo realizado na Dinamarca, pacientes que receberam apoio de um nutricionista ou que participaram de pesquisas sobre a relação entre peso e psoríase consideraram esse acompanhamento altamente benéfico. No entanto, quando esse apoio de outro profissional acabava, o seguimento com o médico especialista tornava-se mais difícil.(22)

Todavia, estudo realizado na Inglaterra demonstrou a importância de os pacientes com psoríase serem acompanhados por médicos especialistas. A pesquisa sugeriu que a prática atual de rotina para o tratamento da psoríase na atenção primária realizada por clínicos é incompatível com as reais necessidades desses pacientes. Assim, para melhoria desta assistência, a psoríase precisaria ser reconhecida como uma condição complexa de longo prazo que envolve demandas físicas, psicológicas e sociais profundas.(29) 
Oferecer cuidados especializados para o restabelecimento da saúde em todos os aspectos, sem se preocupar somente com a integridade da pele, mas também com as questões psicossociais, pode reduzir o impacto negativo da doença na vida dos pacientes.(30)

Consultas de enfermagem e outras ações integradas de saúde também podem contribuir para melhoria da QV, mas é fundamental que as intervenções não se concentrem apenas na doença física. A equipe deve ser sensível aos problemas de saúde e sociais dos pacientes que procuram intervenções alternativas que impactem positivamente.(31)

A consulta de enfermagem é uma estratégia eficaz para melhoria da qualidade de vida do paciente, pois permite ao enfermeiro organizar sistematicamente seus cuidados com o objetivo de prestar cuidados que envolvam não só o aspecto biológico, mas, também, a compreensão do paciente como ser social durante o processo saúde-doença. Tal abordagem permitiria ao enfermeiro fazer parte do processo de identificação de possíveis problemas e decisões a serem tomadas durante o cuidado. (32)

A escassez de estudos na literatura com abordagem qualitativa para investigar a percepção de especialistas sobre o impacto da psoríase na qualidade de vida dos pacientes foi uma limitação do estudo, além de ter sido realizado em um único hospital.

\section{Conclusão}

Os resultados deste estudo demonstram que a psoríase, na perspectiva de dermatologistas, é uma doença que impacta negativamente na qualidade de vida dos pacientes, em virtude do preconceito, dos transtornos psicossociais e sentimentos negativos por ela desencadeados.

Os depoimentos dos profissionais dermatologistas revelaram que a melhora da qualidade de vida desses pacientes perpassa pelo estabelecimento de vínculos com a equipe, assistência humanizada e redução dos sintomas oriundos das lesões cutâneas, com consequente melhora da aparência.

Por fim, a presente análise oferece importantes subsídios aos profissionais de saúde para o aprimoramento das estratégias de cuidado mediante a implementação de ações pautadas em uma abordagem interdisciplinar e integral de atenção à saúde. Faz-se necessário oferecer uma assistência que contemple as reais necessidades psicológicas e sociais desses pacientes, que muitas vezes passam despercebidas no atendimento habitual.

\section{Referências}

1. Fernandes MO, Pereira LFG, Monteiro LVP, Nascimento HJD, Fonseca WLMS da. Efeitos adversos do uso de imunobiológicos no tratamento da psoríase. Rev Med (São Paulo). 2018;97(5):486-92.

2. Sultana A, Bhuiyan SI, Mahmud MM, Siddique RU, Shawkat SM, Nandi AK. Comorbidities in Patients with Psoriasis. Mymensingh Med J. 2019;28(4):894-9.

3. Gonzalez-Andrades M, Arias-Santiago S, García-Serrano JL, González Gallardo M del C, McAlinden C. Sterile Corneal Infiltrates Secondary to Psoriasis Exacerbations: Topical 
Tacrolimus as an Alternative Treatment Option. Eye \& Contact Lens: Science \& Clinical Practice. 2017;43(1):e1-3.

4. Di Meglio P, Villanova F, Nestle FO. Psoriasis. Cold Spring Harbor Perspectives in Medicine. 2014;4(8):a015354-a015354.

5. Kaufman BP, Alexis AF. Psoriasis in Skin of Color: Insights into the Epidemiology, Clinical Presentation, Genetics, Quality-of-Life Impact, and Treatment of Psoriasis in Non-White Racial/Ethnic Groups. Am J Clin Dermatol. 2018;19(3):405-23.

6. Silveira, Maria Eugênia de Bona, Pelegrina Neto, Gabriel, Ferreira, Flávia Regina. Perfil epidemiológico e qualidade de vida na psoríase. 2017;15(4):246-51.

7. Martins GA, Arruda L, Mugnaini ASB. Validação de questionários de avaliação da qualidade de vida em pacientes de psoríase. An Bras Dermatol. 2004;79(5):521-35.

8. The World Health Organization Quality of Life assessment (WHOQOL): position paper from the World Health Organization. Soc Sci Med. 1995;41(10):1403-9.

9. Sawada NO, Nicolussi AC, Paula JM de, Garcia-Caro MP, Marti-Garcia C, Cruz-Quintana F. Quality of life of Brazilian and Spanish cancer patients undergoing chemotherapy: an integrative literature review. Rev Latino-Am Enfermagem [Internet]. 2016 [citado 11 de fevereiro de 2021];24(0). Disponível em: http://www.scielo.br/scielo.php?script=sci_arttext\&pid=S0104$11692016000100601 \& \operatorname{lng}=$ en\&tlng=en

10. Ribeiro de Jesus PB, Santos I dos, Brandão E da S. A autoimagem e a autoestima das pessoas com transtornos de pele: uma revisão integrativa da literatura baseada no modelo de Callista Roy. Aquichan. 2015;15(1):75-89.

11. Pereira ER, Arruda CS, Nogueira E, Oliveira MSD, Silva RMCRA. Avanços e desafios da enfermagem na produção científica sobre psoríase. 2015 [citado 11 de fevereiro de 2021]; Disponível em: http://rgdoi.net/10.13140/RG.2.1.2177.7364

12. Pereira DN, Tolentino GP, Soares V, Venâncio PEM. Qualidade de vida de pessoas com doenças crônicas. Cinergis. 2017;18(3):216.

13. Oliveira-Campos M, Rodrigues-Neto JF, Silveira MF, Neves DMR, Vilhena JM, Oliveira JF, et al. Impacto dos fatores de risco para doenças crônicas não transmissíveis na qualidade de vida. Ciênc saúde coletiva. 2013;18(3):873-82.

14. Melo MSB de, Rocha NFL, Magalhães SS, Sousa LL. Influência de fatores emocionais nas doenças crônicas de pele: $\mathrm{O}$ estresse como gatilho para o desenvolvimento, reincidência ou agravamento da psoríase. 2019;13(46):584-608.

15. Pollo CF, Miot LDB, Meneguin S, Miot HA. Factors associated with quality of life in facial melasma: a cross-sectional study. Int J Cosmet Sci. 7 de maio de 2018. doi: 10.1111/ics.12464

16. Lefevre F, Lefevre AMC, Marques MC da C. Discurso do sujeito coletivo, complexidade e auto-organização. Ciênc saúde coletiva. agosto de 2009;14(4):1193-204.

17. Figueiredo, Marília Z. A, Chiari, Brasilia M, Goulart, Bárbara N. G. de. Discourse of Collective Subject: a brief introduction to a qualitative-quantitative research tool. Distúrb comun [Internet]. 2013;25(1). Disponível em: https://pesquisa.bvsalud.org/portal/resource/pt/lil-673869

18. Brasil. Ministério de Saúde. Conselho Nacional de Saúde.Brasil. Ministério de Saúde. Conselho Nacional de Saúde. Resolução 466 de 12 de dezembro de 2012. Resolução 466 de 12 de dezembro de 2012. 2012. 
19. Cranenburgh O, Smets E, Rie M, Sprangers M, Korte J. A Web-based, Educational, Qualityof-life Intervention for Patients with a Chronic Skin Disease: Feasibility and Acceptance in Routine Dermatological Practice. Acta Derm Venerol. 2015;95(1):51-6.

20. For the PROMPT Study Group, Dures E, Hewlett S, Lord J, Bowen C, McHugh N, et al. Important Treatment Outcomes for Patients with Psoriatic Arthritis: A Multisite Qualitative Study. Patient. agosto de 2017;10(4):455-62.

21. Volz PM, Tomasi E, Bruck NRV, Saes M de O, Nunes BP, Duro SMS, et al. A inclusão social pelo trabalho no processo de minimização do estigma social pela doença. Saude soc. 2015;24(3):877-86.

22. Khoury LR, Skov L, Møller T. Facing the dilemma of patient-centred psoriasis care: a qualitative study identifying patient needs in dermatological outpatient clinics. Br J Dermatol. 2017;177(2):436-44.

23. Tribó M, Turroja M, Castaño-Vinyals G, Bulbena A, Ros E, García-Martínez P, et al. Patients with Moderate to Severe Psoriasis Associate with Higher Risk of Depression and Anxiety Symptoms: Results of a Multivariate Study of 300 Spanish Individuals with Psoriasis. Acta Derm Venerol. 2019;99(4):417-22.

24. Augustin M, Krüger K, Radtke MA, Schwippl I, Reich K. Disease Severity, Quality of Life and Health Care in Plaque-Type Psoriasis: A Multicenter Cross-Sectional Study in Germany. Dermatology. 2008;216(4):366-72.

25. Melo MSB de, Rocha NFL, Magalhães SS, Sousa LL. Influência de fatores emocionais nas doenças crônicas de pele: $\mathrm{O}$ estresse como gatilho para o desenvolvimento, reincidência ou agravamento da psoríase. 2019;13(46):584-608.

26. Fernandes MO, Pereira LFG, Monteiro LVP, Nascimento HJD, Fonseca WLMS da. Efeitos adversos do uso de imunobiológicos no tratamento da psoríase. Rev Med (São Paulo). 2018;97(5):486-92.

27. Mota LMH da, Cruz BA, Brenol CV, Pollak DF, Pinheiro G da RC, Laurindo IMM, et al. Segurança do uso de terapias biológicas para o tratamento de artrite reumatoide e espondiloartrites. Revista Brasileira de Reumatologia. 2015;55(3):281-309.

28. Organização mundial da saúde. Relatório global sobre a psoríase. [Internet]. 2016 [citado 11 de fevereiro de 2021]. Disponível em: https://apps.who.int/iris/bitstream/handle/10665/204417/9789241565189por.pdf? sequence $=17 \&$ is Allowed $=\mathrm{y}$

29. Nelson PA, Barker Z, Griffiths CE, Cordingley L, Chew-Graham CA. 'On the surface': a qualitative study of GPs' and patients' perspectives on psoriasis. BMC Fam Pract. 2013;14(1):158.

30. Guerreiro TN, Rodrigues ILA, Nogueira LMV, Távora MM. Alterações no cotidiano de pessoas acometidas por psoríase. Rev enferm UERJ. 2018;26:e28332.

31. Quental LLC, Nascimento LCC da C, Leal LC, Davim RMB, Cunha ICBC. Práticas educativas com gestantes na atenção primária à saúde. Rev enferm UFPE on line. 2017;11(12):5370.

32. Oliveira SKP de, Queiroz APO, Matos DP de M, Moura AF de, Lima FET. Temas abordados na consulta de enfermagem: revisão integrativa da literatura. Rev Bras Enferm. 2012;65(1):155-161. 
Como citar: De Souza, J. M., De Godoy, N. A., Fernandes Pollo, C., Miot, H.A, Meneguin, S. Percepção de dermatologistas sobre a influência da psoríase na qualidade de vida dos pacientes. Enfermería: $\quad$ Cuidados $\quad$ Humanizados. $\quad 2021 ; 10(1): \quad 18-29 . \quad$ Doi: https://doi.org/10.22235/ech.v10i1.2066

Participação dos autores: a) Planejamento e concepção do trabalho; b) Coleta de dados; c) Análise e interpretação de dados; d) Redação do manuscrito; e) Revisão crítica do manuscrito. J.M.D.S. contribuiu em a, b, c, d,e; N. A. D. G. em a,b,c,d; C. F. P. em a, b,c,d,e.; H. A. M. em a, c,d,e; S. M. em a, b, c, d, e.

Editora científica responsável: Dra. Natalie Figueredo 